\section{Subestimativa de obesidade e sobrepeso a partir de medidas autorrelatadas na população geral: prevalência e proposta de modelos para correção}

\section{Underestimation of obesity and overweight based on self-report measures in the general population: prevalence and a proposal for correction models}

\section{Infravaloración de obesidad y sobrepeso a partirde medidas autoinformadas en población general: prevalencia y propuesta de modelos para su corrección}

\section{Resumo}

Os objetivos deste artigo são: (i) comparar medidas autorrelatadas de peso e estatura com medidas aferidas; (ii) avaliar o impacto dessas discrepâncias sobre o indice de massa corporal (IMC) e as prevalências de sobrepeso e obesidade; e (iii) aplicar modelos de correção das medidas autorrelatadas e avaliar o grau de melhoria das medidas corrigidas produzidas pelo uso desses modelos. Realizou-se estudo transversal, com avaliação de 4.151 adultos (18 a 60 anos) participantes do Estudo Epidemiológico dos Transtornos Mentais São Paulo Megacity. Foram propostos e testados modelos de regressão linear estratificados por sexo, para a correção das medidas autorrelatadas. Para avaliar a concordância, usou-se o coeficiente de correlação intraclasse para as medidas aferidas, medidas autorrelatadas e medidas corrigidas, bem como o coeficiente kappa para as categorias de classificação do IMC. O peso autorrelatado e o IMC resultantes foram subestimados, ao passo que a estatura foi superestimada, comparados às medidas aferidas. Com todos os modelos de correção, as medidas corrigidas tornaram-se mais próximas às medidas aferidas. As prevalências de excesso de peso, quando calculadas a partir das medidas autorrelatadas, estavam subestimadas em 24\% em homens e 28\% em mulheres. Com as correções, a subestimativa diminuiu para $8 \%$ e $10 \%$, respectivamente. Identificou-se concordância moderada para as medidas autorrelatadas e concordância substancial para as medidas corrigidas, quando comparadas às medidas reais. O uso de equações de correção para dados autorrelatados mostrou-se um método útil para produzir estimativas mais fidedignas da prevalência de excesso de peso e obesidade na população geral, geralmente estimadas a partir de medidas de peso e estatura autorrelatadas nos inquéritos populacionais.

Autorrelato; Obesidade; Sobrepeso; Prevalência; Índice de Massa Corporal
Lara Onofre Ferriani 1

Evandro Silva Freire Coutinho 2

Daniela Alves Silva 1

Carolina Perim de Faria 1

Maria del Carmen Bisi Molina 1

Isabela Judith Martins Benseñor 3

Maria Carmen Viana 1

doi: $10.1590 / 0102-311 \times 00065618$

Correspondência

L. O. Ferriani

Universidade Federal do Espirito Santo.

Av. Marechal Campos 1468, Vitória, ES 29040-090, Brasil.

laraonofref@gmail.com

1 Universidade Federal do Espirito Santo, Vitória, Brasil.

2 Fundação Oswaldo Cruz, Rio de Janeiro, Brasil.

3 Universidade de São Paulo, São Paulo, Brasil. 


\section{Introdução}

A obesidade atingiu proporções pandêmicas e é considerada pela Organização Mundial da Saúde (OMS) 1,2, desde o início deste século, um dos principais problemas de saúde pública. Está diretamente associada ao aumento da mortalidade por todas as causas 3 e impacta de forma negativa a autoestima e a qualidade de vida das pessoas afetadas 4 .

A necessidade de monitoramento contínuo do estado nutricional da população vem sendo globalmente preconizada pela OMS 1,2. Nesse contexto, as medidas antropométricas de peso e estatura são utilizadas para calcular o índice de massa corporal (IMC), utilizado e recomendado mundialmente para o diagnóstico do excesso de peso em todos os grupos etários 5 . Informações de peso e de estatura podem ser obtidas por meio da aferição e do autorrelato. Em estudos epidemiológicos, o autorrelato é mais frequentemente utilizado, por facilitar a obtenção dos dados, reduzir os custos e abreviar o tempo de execução 6 .

Estudos anteriores demonstraram que os dados autorrelatados estão fortemente correlacionados com os aferidos 7,8, supondo-se, assim, que o seu uso seja válido. Entretanto, são encontrados erros sistemáticos e discrepâncias nos valores autorrelatados, quando comparados às medidas aferidas, uma vez que o autorrelato mostra-se influenciado por diversas características pessoais, como sexo, idade, estado nutricional, renda e escolaridade, além de padrões estéticos de imagem corporal vigentes nas diferentes culturas 9,10,11,12. Observa-se uma tendência de subestimação do peso e superestimação da estatura, como verificado por Gorber et al. ${ }^{13}$, ao realizarem uma revisão sistemática com as evidências empíricas sobre a concordância entre as medidas autorrelatadas de peso e estatura.

A subestimação do peso e a superestimação da estatura incorrem em uma subestimativa do IMC e, consequentemente, influenciam a classificação do estado nutricional, o que resulta em prevalências subestimadas de sobrepeso e obesidade, bem como em avaliação incorreta do risco de morbimortalidade 14. Um estudo realizado com adultos na Suécia, cujo objetivo foi validar medidas autorrelatadas de peso e estatura, verificou que a prevalência de obesidade, avaliada pelo IMC resultante de dados autorrelatados, foi aproximadamente $5 \%$ menor, tanto em homens quanto em mulheres 15 . Com base nessa constatação, alguns estudos vêm sugerindo métodos de correção dos dados autorrelatados, com o intuito de tornar mais fidedigna a avaliação do estado nutricional da população geral 15,16,17.

Assim, os objetivos deste estudo foram: (i) comparar medidas autorrelatadas de peso e estatura com medidas obtidas por mensuração direta em uma amostra da população geral de adultos; (ii) avaliar o impacto dessas discrepâncias sobre o IMC e as prevalências de sobrepeso e obesidade; e (iii) aplicar métodos de correção das medidas autorrelatadas e avaliar o grau de melhoria das medidas produzidas pelo uso desses métodos.

\section{Métodos}

Os dados analisados neste estudo são provenientes do Estudo Epidemiológico dos Transtornos Mentais São Paulo Megacity (SPMC). Trata-se de um estudo transversal de base populacional que avaliou a morbidade psiquiátrica em uma amostra probabilística da população geral com 18 anos ou mais, residente na Região Metropolitana de São Paulo 18,19,20.

A coleta de dados foi dividida em duas fases: a primeira consistiu em entrevista domiciliar, realizada por entrevistadores leigos treinados, entre maio de 2005 e abril de 2007, na qual as medidas de peso e estatura foram autorrelatadas pelos participantes 18 . Na segunda fase, uma subamostra dos indivíduos entrevistados nos domicílios, com idade entre 18 e 60 anos, foi convidada a participar de múltiplas avaliações no âmbito hospitalar, quando as medidas de peso e estatura foram sistematicamente aferidas com a utilização de técnicas padronizadas por avaliadores treinados 21 .

A amostra populacional foi selecionada por meio de um processo probabilístico multiestratificado, sem substituição, totalizando 5.037 indivíduos entrevistados nos domicílios 18, sendo incluídos nesta análise todos aqueles com idade entre 18 e 60 anos $(n=4.151)$. A coleta de dados da fase hospitalar avaliou uma subamostra de 766 respondentes 21 , dos quais foram excluídas 27 mulheres por não haver informação de peso e/ou estatura na fase domiciliar, o que impossibilitou o cálculo do IMC a 
partir das medidas autorrelatadas. Assim, na análise de medidas aferidas, foi incluído um total de 739 respondentes que tinham a informação de peso e estatura.

A avaliação hospitalar ocorreu no Instituto de Psiquiatria da Faculdade de Medicina da Universidade de São Paulo (FMUSP), onde participantes foram pesados e medidos descalços e com roupas leves, de acordo com as técnicas recomendadas pelo Centro de Controle e Prevenção de Doenças dos Estados Unidos (CDC) 22. Foi realizada a aferição do peso com balança antropométrica digital Toledo, capacidade de pesagem de até $200 \mathrm{~kg}$ (Toledo do Brasil Indústria de Balanças Ltda., São Bernardo do Campo, Brasil), e, para a medida da estatura, utilizou-se estadiômetro acoplado à balança antropométrica (régua medindo de $1 \mathrm{~m}$ a $2 \mathrm{~m}$, com escala de $0,5 \mathrm{~cm}$ ).

O IMC foi calculado dividindo-se o peso em quilogramas pelo quadrado da estatura em metros, e o estado nutricional foi classificado, de acordo com a OMS 5 , em baixo peso $\left(\leq 18,49 \mathrm{~kg} / \mathrm{m}^{2}\right)$, eutrofia $\left(18,5-24,99 \mathrm{~kg} / \mathrm{m}^{2}\right)$, sobrepeso $\left(25,0-29,99 \mathrm{~kg} / \mathrm{m}^{2}\right)$, obesidade grau $1\left(30,0-34,99 \mathrm{~kg} / \mathrm{m}^{2}\right)$, obesidade grau $2\left(35,0-39,99 \mathrm{~kg} / \mathrm{m}^{2}\right)$ e obesidade grau $3\left(\geq 40,0 \mathrm{~kg} / \mathrm{m}^{2}\right)$.

Pesos, estaturas e IMC corrigidos foram obtidos por meio de dois conjuntos de modelos de regressão linear, em que o peso aferido (Modelos P) e a estatura aferida (Modelos E) foram incluídos como variáveis dependentes. Como essas três variáveis apresentavam distribuições desviadas para a direita, utilizamos a transformação logarítmica, sugerida pela rotina "ladder" do programa Stata (https:// www.stata.com), com o intuito de aproximá-las da distribuição normal. Análises exploratórias mostraram que as medidas autorrelatadas, a idade e a escolaridade eram preditoras do peso e da estatura aferidas, tendo sido avaliadas, assim, como variáveis independentes nos modelos para obtenção de fórmulas de correção. Como os dados foram estratificados por sexo, foram avaliados nove modelos para o sexo masculino e nove modelos para o sexo feminino:

- Modelo P1: ajustado por dado autorrelatado;

- Modelo P2: ajustado por dado autorrelatado e idade;

- Modelo P3: ajustado por dado autorrelatado, idade e escolaridade.

- Modelo E1: ajustado por dado autorrelatado;

- Modelo E2: ajustado por dado autorrelatado e idade;

- Modelo E3: ajustado por dado autorrelatado, idade e escolaridade.

- Modelo IMC1: ajustado por dado autorrelatado;

- Modelo IMC2: ajustado por dado autorrelatado e idade;

- Modelo IMC3: ajustado por dado autorrelatado, idade e escolaridade.

Além disso, o IMC também foi corrigido indiretamente ("metacorreção"), com modelos usando as medidas corrigidas de peso e estatura, também estratificados por sexo:

- IMC Metacorrigido 1: utilizando peso e estatura corrigidos pelos Modelos P1 e E1;

- IMC Metacorrigido 2: utilizando peso e estatura corrigidos pelos Modelos P2 e E2;

- IMC Metacorrigido 3: utilizando peso e estatura corrigidos pelos Modelos P3 e E3.

O diagnóstico dos modelos finais foi feito por meio da avaliação da distribuição dos resíduos (gráficos com estimativas de densidade de Kernel), da presença de homocedasticidade dos resíduos (por meio de gráfico e do teste de heterocedasticidade de Breusch-Pagan/Cook-Weisberg) e ausência de multicolinearidade (fator de inflação da variância).

Foram calculados o coeficiente de correlação intraclasse (CCI) e o intervalo de $95 \%$ de confiança (IC95\%) para a avaliação da concordância entre as medidas aferidas de peso, estatura e IMC, e cada uma dessas medidas foi corrigida a partir dos diferentes modelos. Já a concordância entre as categorias de classificação do IMC calculado a partir das medidas aferidas e dos IMC corrigidos por cada modelo foi avaliada pelo coeficiente kappa, sem ponderação, com base na classificação de Landis \& Koch 23.

Todas as análises foram conduzidas no programa Stata, e naquelas em que foram produzidas estimativas populacionais, com o banco domiciliar $(\mathrm{n}=4.151)$, foi usado o modo survey do Stata para amostras com desenhos complexos. Utilizaram-se pesos ajustados para a probabilidade de seleção em todas as etapas de amostragem e para a composição demográfica da população-alvo, por sexo e idade, no Censo Demográfico de 200024.

O estudo SPMC foi aprovado pelo Comitê de Ética e Pesquisa da FMUSP (processo no 792/03). Todos os participantes assinaram o Termo de Consentimento Livre e Esclarecido. 


\section{Resultados}

Observou-se uma subestimação do peso autorrelatado de mais de $1 \mathrm{~kg}$ para ambos os sexos $(1,1 \mathrm{~kg}$ entre homens e $1,4 \mathrm{~kg}$ entre mulheres), quando comparado com o peso aferido. Independente do modelo utilizado para a correção do peso, a média da medida corrigida se apresentou bem próxima à da medida aferida. Já a estatura autorrelatada foi superestimada tanto por homens $(2,4 \mathrm{~cm})$ quanto por mulheres $(3,6 \mathrm{~cm})$. As médias das estimativas corrigidas da estatura autorrelatada mostram-se bastante próximas à da estatura aferida, em todos os modelos de correção propostos (Tabela 1).

O IMC segue o mesmo padrão do peso, ou seja, é subestimado quando calculado a partir das medidas autorrelatadas de peso e estatura, tanto em homens $\left(1,1 \mathrm{~kg} / \mathrm{m}^{2}\right)$ quanto em mulheres $\left(1,6 \mathrm{~kg} / \mathrm{m}^{2}\right)$. Para ambos os sexos, o IMC corrigido diretamente por qualquer um dos três modelos apresentou um valor bem próximo ao IMC obtido a partir do peso e da estatura aferidos. O mesmo ocorreu no caso do IMC metacorrigido, isto é, calculado a partir dos pesos e estaturas corrigidos pelos três modelos (Tabela 1).

Ao se calcular o CCI entre todos os valores corrigidos pelos três modelos e os valores aferidos, verificou-se que, para a medida de peso, a concordância foi praticamente a mesma, independente do modelo de correção, sendo um pouco maior para o sexo masculino. Com relação à estatura, houve melhora da concordância, à proporção que mais variáveis de ajuste eram incluídas no modelo de correção. No caso do IMC, os dois processos de correção apresentaram CCI muito próximos (Tabela 2).

Após a correção das medidas de peso, estatura e IMC pelos diferentes modelos propostos, verificou-se que o ajuste por idade e escolaridade contribuiu pouco para a melhoria das medidas em ambos os sexos, tanto em relação à precisão de cada uma delas, quanto à concordância com as correspondentes medidas aferidas. Apesar disso, considerando que esses resultados podem servir de base para correções em outros estudos populacionais, decidiu-se manter os modelos ajustados pela idade (Modelo 2), uma vez que a fidedignidade dessa variável é bastante elevada, e ela geralmente está disponível em estudos populacionais.

Assim, os algoritmos de correção escolhidos para serem utilizados no cálculo das medidas corrigidas são descritos no Quadro 1.

Ao se verificar a distribuição de acordo com as categorias de estado nutricional, calculadas a partir dos dados aferidos, autorrelatados e corrigidos pelo Modelo 2 na subamostra $(n=766)$, observou-se uma superestimativa na categoria baixo peso e eutrofia e também uma subestimativa de excesso de peso, ao serem comparadas as medidas aferidas e autorrelatadas, em ambos os sexos. De um modo geral, essas discrepâncias foram amenizadas com a classificação de estado nutricional a partir dos IMC corrigidos. No entanto, a distribuição mostrou-se mais inconsistente nos dois extremos das categorias de classificação, possivelmente devido à prevalência reduzida de indivíduos com baixo peso e obesidade grau 3, como também à consequente perda de precisão para a correção a partir dos modelos de regressão linear (Tabela 3 ).

Em respondentes de ambos os sexos, as estimativas de sobrepeso e obesidade com base nas medidas aferidas são superiores àquelas obtidas com base no autorrelato. Ao analisar separadamente as prevalências de sobrepeso calculadas a partir dos dados autorrelatados em comparação com os aferidos, observa-se uma subestimativa de $13 \%$ nos homens e de $18 \%$ nas mulheres. Com as correções, as prevalências de sobrepeso ficaram mais próximas. A prevalência de obesidade por meio dos dados autorrelatados também foi subestimada em cerca de $40 \%$ em ambos os sexos. Com as correções, as estimativas de obesidade também ficaram mais próximas das estimativas reais (Tabela 4).

As prevalências de excesso de peso (sobrepeso e obesidade graus 1, 2 e 3), quando calculadas a partir dos dados autorrelatados em comparação com os dados aferidos, são subestimadas em $24 \%$ entre os homens e $28 \%$ entre as mulheres. Verificou-se que, com as correções, essa subestimativa foi reduzida para 11\% e $8 \%$ (Corrigido 2) e 12\% e 10\% (Metacorrigido 2), respectivamente (Tabela 4).

Apesar de discrepâncias entre as prevalências de sobrepeso e obesidade permanecerem, mesmo com a correção das medidas autorrelatadas, pode-se verificar que a concordância das estimativas corrigidas com as medidas reais é melhor, tanto para homens quanto para mulheres, com coeficientes de kappa mostrando concordância moderada para as medidas autorrelatadas e concordância substancial para as medidas corrigidas (Tabela 4). 


\section{Tabela 1}

Média e desvio padrão (DP) de peso, estatura e índice de massa corporal (IMC) calculados a partir das medidas aferidas, autorrelatadas e corrigidas pelos diferentes modelos $(n=766)$.

\begin{tabular}{|c|c|c|c|c|c|}
\hline & \multicolumn{5}{|c|}{ Peso (kg) } \\
\hline & Aferido & Autorrelatado & Corrigido 1 * & Corrigido 2 ** & Corrigido $3 * * *$ \\
\hline \multicolumn{6}{|l|}{ Masculino } \\
\hline Média & 78,58 & 77,48 & 78,48 & 78,48 & 78,48 \\
\hline $\mathrm{DP}$ & 15,48 & 14,45 & 15,43 & 15,44 & 15,44 \\
\hline \multicolumn{6}{|l|}{ Feminino } \\
\hline Média & 66,91 & 65,47 & 66,76 & 66,76 & 66,76 \\
\hline \multirow[t]{3}{*}{ DP } & 14,31 & 13,11 & 14,17 & 14,17 & 14,18 \\
\hline & \multicolumn{5}{|c|}{ Estatura $(\mathrm{cm})$} \\
\hline & Aferida & Autorrelatada & Corrigida 1 * & Corrigida 2 ** & Corrigida $3 * * *$ \\
\hline \multicolumn{6}{|l|}{ Masculino } \\
\hline Média & 170,06 & 172,45 & 170,04 & 170,03 & 170,04 \\
\hline DP & 7,18 & 7,69 & 6,39 & 6,40 & 6,43 \\
\hline \multicolumn{6}{|l|}{ Feminino } \\
\hline Média & 157,35 & 160,94 & 157,53 & 157,53 & 157,53 \\
\hline \multirow[t]{3}{*}{ DP } & 6,13 & 6,44 & 4,98 & 5,04 & 5,09 \\
\hline & \multicolumn{5}{|c|}{ IMC (kg/m²) } \\
\hline & Aferido & Autorrelatado & Corrigido 1 * & Corrigido 2 ** & Corrigido $3 * * *$ \\
\hline \multicolumn{6}{|l|}{ Masculino } \\
\hline Média & 27,09 & 25,99 & 27,01 & 27,01 & 27,01 \\
\hline DP & 4,55 & 4,16 & 4,33 & 4,33 & 4,33 \\
\hline \multicolumn{6}{|l|}{ Feminino } \\
\hline Média & 26,99 & 25,38 & 26,95 & 26,95 & 26,95 \\
\hline \multirow[t]{3}{*}{ DP } & 5,35 & 4,80 & 4,94 & 4,93 & 4,93 \\
\hline & \multicolumn{5}{|c|}{ IMC (kg/m²) } \\
\hline & Aferido & Autorrelatado & Metacorrigido 1 \# & Metacorrigido $2 \# \#$ & Metacorrigido 3 \#\#\# \\
\hline \multicolumn{6}{|c|}{ Masculino } \\
\hline Média & 27,09 & 25,99 & 27,06 & 27,06 & 27,09 \\
\hline DP & 4,55 & 4,16 & 4,56 & 4,58 & 4,58 \\
\hline \multicolumn{6}{|l|}{ Feminino } \\
\hline Média & 26,99 & 25,38 & 26,98 & 26,99 & 27,00 \\
\hline DP & 5,35 & 4,80 & 5,38 & 5,43 & 5,43 \\
\hline
\end{tabular}

* Calculado a partir do Modelo 1;

** Calculado a partir do Modelo 2;

*** Calculado a partir do Modelo 3;

\# Calculado a partir das medidas de peso e estatura corrigidas pelo Modelo 1 ;

\#\# Calculado a partir das medidas de peso e estatura corrigidas pelo Modelo 2;

\#\# Calculado a partir das medidas de peso e estatura corrigidas pelo Modelo 3.

Observou-se uma prevalência de excesso de peso de 42,6\%, ao calcular o IMC a partir dos dados autorrelatados e classificar o estado nutricional da população adulta (18-60 anos) residente na Região Metropolitana de São Paulo. Ao aplicar os modelos de correção, essa estimativa aumentou para 52,8\% (Corrigido 2) e 52,3\% (Metacorrigido 2), com uma subestimativa do excesso de peso populacional de $20 \%$ e 19\%, respectivamente (Tabela 5). A prevalência de excesso de peso com base no autorrelato é maior entre homens do que entre mulheres $(48,1 \%$ vs. $37,3 \%)$, mas a subestimativa foi maior entre as 


\section{Tabela 2}

Coeficientes de correlação intraclasse (CCI) e intervalos de 95\% de confiança (IC95\%) entre as medidas aferidas e as medidas corrigidas de peso, estatura e índice de massa corporal (IMC), e metacorrigidas de IMC ( $\mathrm{n}=766)$.

\begin{tabular}{|c|c|c|}
\hline & $\begin{array}{l}\text { Masculino } \\
\text { CCI (IC95\%) }\end{array}$ & $\begin{array}{l}\text { Feminino } \\
\text { CCI (IC95\%) }\end{array}$ \\
\hline \multicolumn{3}{|l|}{ Peso } \\
\hline Aferido $x$ corrigido 1 * & $0,948(0,938-0,959)$ & $0,933(0,920-0,945)$ \\
\hline Aferido $x$ corrigido $2 * \star$ & $0,950(0,938-0,959)$ & $0,934(0,920-0,945)$ \\
\hline Aferido $x$ corrigido $3 * \star *$ & $0,950(0,938-0,959)$ & $0,934(0,921-0,945)$ \\
\hline \multicolumn{3}{|l|}{ Estatura } \\
\hline Aferida $x$ corrigida 1 * & $0,881(0,855-0,903)$ & $0,802(0,764-0,834)$ \\
\hline Aferida $x$ corrigida $2 * \star$ & $0,882(0,856-0,904)$ & $0,811(0,776-0,842)$ \\
\hline Aferida $\times$ corrigida $3 * \star \star$ & $0,886(0,861-0,907)$ & $0,823(0,789-0,852)$ \\
\hline \multicolumn{3}{|l|}{ IMC } \\
\hline Aferido $x$ corrigido 1 * & $0,903(0,881-0,921)$ & $0,896(0,875-0,914)$ \\
\hline Aferido $x$ corrigido 2 ** & $0,914(0,894-0,930)$ & $0,896(0,876-0,914)$ \\
\hline Aferido $x$ corrigido $3 * \star \star$ & $0,903(0,881-0,921)$ & $0,897(0,876-0,914)$ \\
\hline Aferido x metacorrigido 1 \# & $0,909(0,888-0,926)$ & $0,900(0,880-0,917)$ \\
\hline Aferido $\times$ metacorrigido $2 \# \#$ & $0,910(0,888-0,923)$ & $0,901(0,881-0,918)$ \\
\hline Aferido x metacorrigido $3 \# \# \#$ & $0,910(0,890-0,927)$ & $0,910(0,881-0,918)$ \\
\hline
\end{tabular}

* Calculado a partir do Modelo 1;

** Calculado a partir do Modelo 2;

*** Calculado a partir do Modelo 3;

\# Calculado a partir das medidas de peso e estatura corrigidas pelo Modelo 1;

\#\# Calculado a partir das medidas de peso e estatura corrigidas pelo Modelo 2;

\#\#\# Calculado a partir das medidas de peso e estatura corrigidas pelo Modelo 3.

\section{Quadro 1}

Algoritmos para a correção das medidas autorrelatadas pelo Modelo 2 (equações de regressão linear).

\begin{tabular}{|c|}
\hline Sexo masculino \\
\hline $\log$ do peso corrigido $=3,366357+(0,0127014 \times$ peso autorrelatado $)+(-0,0001286 \times$ idade $)$ \\
\hline $\log$ da estatura corrigida $=4,312546+(0,004815 \times$ estatura autorrelatada $)+(-0,000191 \times$ idade $)$ \\
\hline Log do IMC corrigido = 2,334209 + (0,0364284 x IMC autorrelatado $)+(0,0000858 \times$ idade $)$ \\
\hline $\mathrm{IMC}$ metacorrigido $=\log$ do peso corrigido $/ \log$ da estatura corrigida 2 \\
\hline Sexo feminino \\
\hline $\log$ do peso corrigido $=3,229253+(0.0146013 \times$ peso autorrelatado $)+(-0,0000883 \times$ idade $)$ \\
\hline $\log$ da estatura corrigida $=4,305512+(0,0047845 \times$ estatura autorrelatada $)+(-0,0004197 \times$ idade $)$ \\
\hline Log do IMC corrigido = 2,361005 + (0,0351877 x IMC autorrelatado $)+(0,0006319 \times$ idade $)$ \\
\hline IMC metacorrigido $=\log$ do peso corrigido $/ \log$ da estatura corrigida 2 \\
\hline
\end{tabular}

IMC: índice de massa corporal. 


\section{Tabela 3}

Percentual e erro padrão (EP) de acordo com as categorias de estado nutricional obtidas por meio dos índices de massa corporal (IMC) aferidos, autorrelatados e corrigidos $(n=766)$.

\begin{tabular}{lcccc}
\hline IMC & $\begin{array}{c}\text { Aferido } \\
\text { \% (EP) }\end{array}$ & $\begin{array}{c}\text { Autorrelatado } \\
\text { \% (EP) }\end{array}$ & $\begin{array}{c}\text { Corrigido 2 * } \\
\text { \% (EP) }\end{array}$ & $\begin{array}{c}\text { Metacorrigido 2 ** } \\
\text { \% (EP) }\end{array}$ \\
\hline Homens & & & & \\
Baixo peso & $1,2(0,60)$ & $2,5(0,34)$ & $0,2(0,10)$ & $0,4(0,14)$ \\
Eutrofia & $31,9(2,56)$ & $46,6(1,08)$ & $39,9(1,06)$ & $40,9(1,06)$ \\
Sobrepeso & $43,4(2,72)$ & $37,4(1,05)$ & $42,6(1,07)$ & $40,5(1,06)$ \\
Obesidade grau 1 & $16,6(2,04)$ & $10,8(0,67)$ & $12,4(0,71)$ & $13,1(0,73)$ \\
Obesidade grau 2 & $6,6(1,37)$ & $2,3(0,33)$ & $3,6(0,40)$ & $3,6(0,40)$ \\
Obesidade grau 3 & $0,3(0,30)$ & $0,3(0,12)$ & $1,2(0,24)$ & $1,5(0,27)$ \\
Mulheres & & & & \\
Baixo peso & $2,1(0,68)$ & $3,8(0,38)$ & $0,1(0,05)$ & $0,4(0,13)$ \\
Eutrofia & $37,1(2,32)$ & $52,1(0,99)$ & $44,0(0,98)$ & $44,7(0,98)$ \\
Sobrepeso & $36,6(2,31)$ & $29,9(0,90)$ & $37,1(0,95)$ & $34,7(0,94)$ \\
Obesidade grau 1 & $16,1(1,77)$ & $10,1(0,59)$ & $12,6(0,65)$ & $13,8(0,68)$ \\
Obesidade grau 2 & $5,5(1,10)$ & $2,9(0,33)$ & $3,5(0,36)$ & $3,4(0,36)$ \\
Obesidade grau 3 & $2,5(0,75)$ & $1,0(0,20)$ & $2,7(0,32)$ & $3,0(0,34)$ \\
\hline
\end{tabular}

* Calculado a partir do Modelo 2;

** Calculado a partir das medidas de peso e estatura corrigidas pelo Modelo 2.

Tabela 4

Percentual de sobrepeso e obesidade (e excesso de peso) obtidos por meio dos índices de massa corporal (IMC) aferido, autorrelatado e pelos melhores algoritmos de correção do IMC autorrelatado e coeficientes de kappa com respectivos erros padrões (EP) $(n=766)$.

\begin{tabular}{lcccc}
\hline IMC & $\begin{array}{c}\text { Aferido } \\
\text { \% (EP) }\end{array}$ & $\begin{array}{c}\text { Autorrelatado } \\
\text { \% (EP) }\end{array}$ & $\begin{array}{c}\text { Corrigido 2 * } \\
\text { \% (EP) }\end{array}$ & $\begin{array}{c}\text { Metacorrigido 2 ** } \\
\text { \% (EP) }\end{array}$ \\
\hline $\begin{array}{l}\text { Homens } \\
\text { Sobrepeso }\end{array}$ & $43,4(2,72)$ & $37,4(1,05)$ & $42,6(1,07)$ & $40,5(1,06)$ \\
Obesidade & $23,5(2,33)$ & $13,5(0,74)$ & $17,2(0,82)$ & $18,2(0,83)$ \\
Excesso de peso & 66,9 & 50,9 & 59,8 & 58,7 \\
Kappa *** & - & $0,584(0,040)$ & $0,671(0,040)$ & $0,657(0,040)$ \\
Mulheres & & & $37,1(0,95)$ & $34,7(0,94)$ \\
Sobrepeso & $36,6(2,31)$ & $29,9(0,90)$ & $18,8(0,77)$ & $20,2(0,79)$ \\
Obesidade & $24,2(2,06)$ & $14,1(0,69)$ & 55,9 & 54,9 \\
Excesso de peso & 60,8 & 44,0 & $0,638(0,035)$ & $0,657(0,035)$ \\
Kappa *** & - & $0,562(0,035)$ & & \\
\hline
\end{tabular}

* Calculado a partir do Modelo 2;

** Calculado a partir das medidas de peso e estatura corrigidas pelo Modelo 2;

*** Concordância com o dado aferido, considerando 3 categorias: baixo-peso/eutrofia, sobrepeso e obesidade. 
Tabela 5

Prevalências estimadas de eutrofia, sobrepeso e obesidade na população geral residente na Região Metropolitana de São Paulo, Brasil, de 18 a 60 anos, obtidas pelo Modelo 2 de correção $(n=4.151)$.

\begin{tabular}{|c|c|c|c|c|c|c|}
\hline \multirow[t]{2}{*}{ IMC } & \multicolumn{2}{|c|}{ Autorrelatado } & \multicolumn{2}{|c|}{ Corrigido 2 * } & \multicolumn{2}{|c|}{ Metacorrigido 2 ** } \\
\hline & $\%$ & IC95\% & $\%$ & IC95\% & $\%$ & IC95\% \\
\hline \multicolumn{7}{|l|}{ Total } \\
\hline Eutrofia $* \star \star$ & 57,4 & $55,3-59,4$ & 47,2 & $45,1-49,2$ & 47,7 & $45,7-49,7$ \\
\hline Sobrepeso & 30,7 & $29,1-32,4$ & 37,1 & $35,6-38,7$ & 35,3 & $33,9-36,9$ \\
\hline Obesidade & 11,9 & $10,7-13,1$ & 15,7 & $14,4-17,1$ & 17,0 & $15,8-18,2$ \\
\hline \multicolumn{7}{|l|}{ Homens } \\
\hline Eutrofia $* \star \star$ & 51,9 & $49,1-54,6$ & 43,1 & $40,5-45,9$ & 43,7 & $41,0-46,6$ \\
\hline Sobrepeso & 35,5 & $33,3-37,7$ & 40,2 & $37,9-42,4$ & 38,4 & $36,0-40,9$ \\
\hline Obesidade & 12,6 & $11,3-14,2$ & 16,7 & $15,0-18,6$ & 17,9 & $16,1-19,7$ \\
\hline \multicolumn{7}{|l|}{ Mulheres } \\
\hline 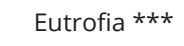 & 62,7 & $58,9-66,3$ & 51,1 & $47,4-54,7$ & 51,5 & $48,0-55,0$ \\
\hline Sobrepeso & 26,2 & $23,4-29,2$ & 34,2 & $31,0-37,6$ & 32,4 & $29,2-35,8$ \\
\hline Obesidade & 11,1 & $9,5-12,9$ & 14,7 & $12,7-17,0$ & 16,1 & $14,1-18,4$ \\
\hline
\end{tabular}

IMC: índice de massa corporal.

* Calculado a partir do Modelo 2;

** Calculado a partir das medidas de peso e estatura corrigidas pelo Modelo 2;

*** Baixo peso + eutrofia.

mulheres (15\% e $15 \%$ vs. $24 \%$ e $24 \%$ ). Portanto, os valores se aproximaram nas estimativas corrigidas de prevalência de excesso de peso, tanto aplicando-se o modelo corrigido 2 (56,9\% vs. 48,9\%), quanto o modelo metacorrigido 2 (56,3\% vs. 48,5\%). Destaca-se que as estimativas de prevalência de obesidade foram semelhantes entre homens e mulheres, o que significa que as mulheres têm proporções maiores de excesso de peso mais grave do que os homens.

\section{Discussão}

Este estudo identificou estimativas elevadas de prevalência de sobrepeso e obesidade na população geral adulta, com idade entre 18 e 60 anos, residente na Região Metropolitana de São Paulo.

Verificou-se uma importante discrepância entre as medidas aferidas e autorrelatadas de peso e estatura, assim como do IMC calculado a partir delas. Como consequência, a utilização de peso e estatura autorrelatados produz falhas na avaliação e classificação do estado nutricional, de acordo com as categorias do IMC, o que resulta em erro sistemático nas estimativas populacionais da prevalência de excesso de peso. Resultados semelhantes foram encontrados em outros estudos 11,13,25, indicando que há um grau significativo de inacurácia na prevalência real de obesidade em estimativas que se utilizam de medidas autorrelatadas.

Muitos estudos recorrem ao uso dessas medidas autorrelatadas para a identificação do risco nutricional em populações. Dentre eles, se destacam os inquéritos nacionais, como o VIGITEL (Vigilância de Fatores de Risco e Proteção para Doenças Crônicas por Inquérito Telefônico), no Brasil 26; o Inquérito Nacional de Saúde, em Portugal 27; e o National Health Interview Surveys, nos Estados Unidos 28. No relatório de 2008, o primeiro publicado após a coleta de dados deste estudo (SPMC), o VIGITEL 29 divulgou uma prevalência de $45,7 \%$ de excesso de peso, sendo $13,8 \%$ de obesidade na população adulta ( $\geq 18$ anos) da cidade de São Paulo. Ao considerar o percentual de subestimativa de excesso de peso encontrado nesse estudo de 19\% (Corrigido 2) e de 19\% (Metacorrigido 2) e de obesidade de 24\% (Corrigido 2) e de 30\% (Metacorrigido 2) e extrapolar esses achados para as estimativas do VIGITEL/SP-2008, a prevalência real de excesso de peso entre adultos brasileiros poderia chegar a 56,4\% (ambos os modelos) e de obesidade atingiria aproximadamente 18,2\% e 19,7\% (Modelo 2 e Metacorreção 2, respectivamente). 
Mais recentemente, em sua última publicação, de 2016, o VIGITEL 30 identificou que 53,4\% dos moradores da cidade de São Paulo tinham excesso de peso, sendo 18,1\% com obesidade. Ao aplicar a correção dessas estimativas, considerando os percentuais de subestimativa encontrados neste estudo, a prevalência real de excesso de peso entre adultos brasileiros poderia chegar a $66,9 \%$, sendo $23,8 \%$ e 25,9\% com obesidade (Modelo 2 e Metacorreção 2, respectivamente).

A subestimativa pode impactar negativamente a implementação de políticas públicas que façam frente ao problema real, desde estratégias globais, como a divulgação de informação e educação da população como medidas preventivas, até a identificação e seguimento de grupos de maior risco, que necessitam de ações específicas. A subestimativa afeta também o planejamento adequado de assistência especializada, incluindo a avaliação e a identificação de doenças associadas à obesidade e de incapacitação resultante, bem como a provisão de tratamentos específicos e reabilitação.

A criação de algoritmos para a correção das medidas autorrelatadas mostrou-se uma alternativa eficaz, pois, apesar de a subestimativa de excesso de peso ainda permanecer com a correção, ela foi reduzida em 13\% (Corrigido 2) e 11\% (Metacorrigido 2) entre os homens, e 19\% (Corrigido 2) e $18 \%$ (Metacorrigido 2) entre as mulheres. Esses achados corroboram outros estudos que utilizaram fórmulas de correção para peso, estatura e IMC autorrelatados 15,16. Entretanto, observa-se que os modelos não foram capazes de corrigir completamente o viés associado ao autorrelato, como também identificado por outros pesquisadores 31,32 . Notaram-se discrepâncias, especialmente nos extremos das categorias de classificação do IMC, que têm menor representação na população geral. No extremo superior, no entanto, o erro é produzido por um comportamento diferente, já que a prevalência de baixo peso mostra-se superestimada, quando utilizadas medidas autorrelatadas 33 .

Este estudo testou diferentes algoritmos de correção com distintas variáveis de ajuste, chegando a resultados corrigidos bastante aproximados. Assim, nas equações de correção, optou-se pela utilização apenas da medida autorrelatada e da idade, já que esta última é uma medida fidedigna e geralmente está disponível em estudos populacionais, permitindo que esses algoritmos de correção possam ser utilizados em estudos de populações brasileiras semelhantes, ou seja, de adultos entre 18 e 60 anos, domiciliados em grandes centros urbanos. É importante enfatizar que todos os modelos foram criados e testados para cada sexo separadamente. Além disso, corrigiu-se o IMC direta e indiretamente (corrigindo o peso e a estatura antes do cálculo e, posteriormente, aplicando-se os algoritmos de correção).

A amostra total desta análise deriva de um estudo transversal de base populacional, representativa da população adulta residente na Região Metropolitana de São Paulo. A subamostra na qual as medidas de peso e estatura foram aferidas tem a mesma distribuição por sexo, faixa etária, escolaridade, renda per capita e prevalência de depressão atual, quando comparada à da amostra total.

Uma possível limitação deste estudo decorre da impossibilidade de estimar o tempo transcorrido entre a fase domiciliar, em que o peso e a estatura foram autorrelatados, e a fase hospitalar, em que essas medidas foram aferidas, podendo ter ocorrido alguma variação no peso, mas não na estatura. O protocolo de pesquisa preconizava reavaliar todos os respondentes em um período de até duas semanas após a avaliação domiciliar, mas não foi possível analisar as datas e estimar a magnitude desse período de tempo. Destaca-se ainda que a utilização do estadiômetro acoplado à balança pode ter gerado algum grau de imprecisão na medida de estatura, reduzindo, assim, a sua confiabilidade.

Por fim, considerando as estimativas decorrentes da aplicação de todas as correções idealizadas e testadas nos diferentes modelos, não houve diferença entre as prevalências observadas, pois a inclusão de duas ou mais variáveis de ajuste não representou efeito significativo sobre os modelos testados. O algoritmo de correção apresentado difere do de outros utilizados por estudos com objetivos semelhantes quanto às variáveis incluídas para ajuste, nos quais também não foi possível corrigir completamente as discrepâncias entre as medidas 15,17,34,35. Ainda assim, as fórmulas de correção são ferramentas apropriadas para estimar prevalências corrigidas, visto que houve incremento na concordância com as medidas aferidas e na aproximação das estimativas com as medidas reais, destacando-se sua aplicabilidade futura em estudos populacionais que utilizem medidas autorrelatadas. Assim, parece importante destacar a frequente subestimativa das prevalências de excesso de peso e obesidade na população geral e aplicar métodos como estes, aqui propostos, para que dados mais fidedignos possam ser estimados, de modo a melhor orientar políticas públicas e ações de prevenção e de enfrentamento desse importante fator de risco para diversos agravos à saúde da população. 


\section{Colaboradores}

L. O. Ferriani contribuiu substancialmente para a concepção e planejamento deste estudo, condução da análise estatística e interpretação dos resultados, elaboração do rascunho do manuscrito, revisão crítica do conteúdo e participação na aprovação da versão final do texto. E. S. F. Coutinho contribuiu substancialmente para a concepção e planejamento deste estudo, condução da análise estatística e interpretação dos resultados, revisão crítica do conteúdo e participação na aprovação da versão final do texto. D. A. Silva, C. P. Faria e M. C. B. Molina contribuiu para a condução da análise estatística e interpretação dos resultados, revisão crítica do conteúdo e participação na aprovação da versão final do manuscrito. I. J. M. Benseñor contribuiu para a realização da fase hospitalar do SPMC, revisão crítica do conteúdo e participação na aprovação da versão final do manuscrito. M. C. Viana contribuiu substancialmente para a concepção e planejamento deste estudo, condução da análise estatística e interpretação dos resultados, revisão crítica do conteúdo, aprovação da versão final, bem como coordenação, concepção e realização do SPMC.

\section{Informações adicionais}

ORCID: Lara Onofre Ferriani (0000-0002-60958590); Evandro Silva Freire Coutinho (0000-00024649-7353); Daniela Alves Silva (0000-0001-73962305); Carolina Perim de Faria (0000-0001-91651332); Maria del Carmen Bisi Molina (0000-00028614-988X); Isabela Judith Martins Benseñor (0000-0002-6723-5678); Maria Carmen Viana (0000-0002-0464-4845).

\section{Agradecimentos}

O Estudo Epidemiológico dos Transtornos Mentais São Paulo Megacity (SPMC) foi apoiado pela Fundação de Amparo à Pesquisa do Estado de São Paulo (FAPESP 03/00204-3), e o desenvolvimento de instrumentos foi apoiado pelo Fundo de Apoio à Ciência e Tecnologia de Vitória (FACITEC 002/2003). O SPMC faz parte de um consórcio da Organização Mundial da Saúde (World Mental Health Survey) e das Universidades de Harvard e Michigan (Estados Unidos).

\section{Referências}

1. World Health Organization. WHO global strategy on diet, physical activity and health: European regional consultation meeting report. Geneva: World Health Organization; 2003.

2. World Health Organization. Obesity: preventing and managinf the global epidemic. Report of a World Health Organization consultation on obesity. Geneva: World Health Organization; 2000. (WHO Technical Report Series, 894).

3. Gonzalez AB, Hartge P, Cerhan JR, Flint AJ, Hannan L, MacInnis RJ, et al. Body-mass index and mortality among 1.46 million white adults. N Engl J Med 2010; 363:2211-9.

4. Kushner RF, Foster GD. Obesity and quality of life. Nutrition 2000; 16:947-52.

5. World Health Organization. Physical status: the use and interpretation of anthropometry. Report of a World Health Organization Expert Committee. Geneva: World Health Organization; 1995. (WHO Technical Report Series, 854).

6. Kac G, Sichieri R, Gigante DP. Epidemiologia nutricional. Rio de Janeiro: Editora Fiocruz/ Ateneu; 2007.

7. Pursey K, Burrows TL, Stanwell P, Collins CE. How accurate is web-based self-reported height, weight, and body mass index in young adults? J Med Internet Res 2014; 16:e4.

8. Conde WL, Oliveira DR, Borges CA, Baraldi LG. Consistência entre medidas antropométricas em inquéritos nacionais. Rev Saúde Pública 2013; 47:69-76.

9. Bolton-Smith C, Woodward M, Tunstall-Pedoe H, Morrison C. Accuracy of the estimated prevalence of obesity from self reported height and weight in an adult Scottish population. J Epidemiol Community Health 2000; 54:143-8.

10. Ramos E, Lopes C, Oliveira A, Barros H. Unawareness of weight and height: the effect on self-reported prevalence of overweight in a population-based study. J Nutr Health Aging 2009; 13:310-4.

11. Danubio ME, Miranda G, Vinciguerra MG Vecchi E, Rufo F. Comparison of self-reported and measured height and weight: implications for obesity research among young adults. Econ Hum Biol 2008; 6:181-90.

12. Hattori A, Sturm R. The obesity epidemic and changes in self-report biases in BMI. Obesity 2013; 21:856-60.

13. Gorber SC, Tremblay M, Moher D, Gorber B. A comparison of direct vs. self-report measures for assessing height, weight and body mass index: a systematic review. Obes Rev 2007; 8:307-26.

14. Scribani M, Shelton J, Chapel D, Krupa N, Wyckoff L, Jenkins P. Comparison of bias resulting from two methods of self-reporting height and weight: a validation study. JRSM Open 2014; 5:2042533313514048. 
15. Nyholm M, Gullberg B, Merlo J, LundqvistPersson C, Råstam L, Lindblad U. The validity of obesity based on self-reported weight and height: implications for population studies. Obesity 2007; 15:197-208.

16. Mozumdar A, Liguori G. Corrective equations to self-reported height and weight for obesity estimates among U.S. adults: NHANES 19992008. Res Q Exerc Sport 2016; 87:47-58.

17. Ellert U, Brettschneider A-K, Wiegand S, Kurth B-M. Applying a correction procedure to the prevalence estimates of overweight and obesity in the German part of the HBSC study. BMC Res Notes 2014; 7:181.

18. Viana MC, Teixeira MG, Beraldi F, Bassani IS, Andrade LH. São Paulo Megacity Mental Health Survey - a population-based epidemiological study of psychiatric morbidity in the São Paulo Metropolitan Area: aims, design and field implementation. Rev Bras Psiquiatr 2009; $31: 375-86$

19. Viana MC, Andrade LH. Lifetime prevalence, age and gender distribution and age-of-onset of psychiatric disorders in the São Paulo Metropolitan Area, Brazil: results from the São Paulo Megacity Mental Health Survey. Rev Bras Psiquiatr 2012; 34:249-60.

20. Andrade LH, Wang Y, Andreoni S, Magalha C, Alexandrino-silva C, Siu ER, et al. Mental disorders in megacities: findings from the São Paulo Megacity Mental Health Survey, Brazil. PLoS One 2012; 7:e31879.

21. Lima DB. Perfil de fatores de risco para doença cardiovascular em amostra de estudo epidemiológico populacional de morbidade psiquiátrica: Estudo São Paulo Megacity [Dissertação de Mestrado]. São Paulo: Faculdade de Medicina, Universidade de São Paulo; 2011.

22. National Center for Health Statistics, Centers for Disease Control and Prevetion. National Health and Nutrition Examination Survey Data. Anthropometry procedures manual. Hyattsville: Centers for Disease Control and Prevention; 2004

23. Landis JR, Koch GG. The measurement of observer agreement for categorical data. Biometrics 1977; 33:159-74.

24. Instituto Brasileiro de Geografia e Estatística. Censo Demográfico 2000: características da população e dos domicílios: resultados do universo. http://www.ibge.gov.br/home/estatis tica/populacao/censo2000/ (acessado em 25/ $\mathrm{Jul} / 2017$ ).
25. Spencer EA, Appleby PN, Davey GK, Key TJ. Validity of self-reported height and weight in 4808 EPIC-Oxford participants. Public Health Nutr 2002; 5:561-5.

26. Ministério da Saúde. Vigitel Brasil 2015 Saúde Suplementar: vigilância de fatores de risco e proteção para doenças crônicas por inquérito telefônico. Brasília: Ministério da Saúde; 2017.

27. Instituto Nacional de Estatística. Inquérito Nacional de Saúde 2014. Edição 2016. Lisboa: Instituto Nacional de Estatística; 2016.

28. National Center for Health Statistics, Centers for Disease Control and Prevetion. Leisuretime physical activity recodes. http://www. cdc.gov/nchs/nhis/physical_activity/pa_re codes.htm (acessado em 25/Jul/2017).

29. Ministério da Saúde. Vigitel Brasil 2008: vigilância de fatores de risco e proteção para doenças crônicas por inquérito telefônico. Brasília: Ministério da Saúde; 2009.

30. Ministério da Saúde. Vigitel Brasil 2016: vigilância de fatores de risco e proteção para doenças crônicas por inquérito telefônico. Brasília: Ministério da Saúde; 2017.

31. Sundquist K, Qvist J, Johansson S-E, Sundquist $\mathrm{J}$. Increasing trends of obesity in Sweden between 1996/97 and 2000/01. Int J Obes Relat Metab Disord 2004; 28:254-61.

32. Plankey MW, Stevens J, Flegal KM, Rust PF. Prediction equations do not eliminate systematic error in self-reported body mass index. Obes Res 1997; 5:308-14.

33. Peixoto MRG, Benício MHD, Jardim PCBV. Validade do peso e da estatura auto-referidos: o estudo de Goiânia. Rev Saúde Pública 2006; 40:1065-72.

34. Brettschneider AK, Schaffrath Rosario A, Wiegand S, Kollock M, Ellert U. Development and validation of correction formulas for self-reported height and weight to estimate BMI in adolescents. Results from the KiGGS Study. Obes Facts 2015; 8:30-42.

35. Santos O, Carmo I, Camolas J, Vieira J. Validade do auto-relato do peso e da estatura na avaliação do índice de massa corporal da população adulta portuguesa. Endocrinologia, Diabetes \& Obesidade 2009; 3:157-68. 


\section{Abstract}

The study's objectives: compare self-report measures of weight and height with direct measures; assess the impact of these discrepancies on body mass index (BMI) and prevalence of overweight and obesity; and apply correction models to the self-report measures and assess the degree of improvement in the corrected measures produced with the use of these models. A cross-sectional study was performed, assessing 4,151 adults (18 to 60 years) participating in the São Paulo Megacity Epidemiological Study. Linear regression models stratified by sex were proposed for correction of self-reported measures. Agreement was assessed with the intraclass correlation coefficient for the direct measures, self-report measures, and corrected measures, and kappa coefficient for BMI classification categories. Self-reported weight and the resulting BMI were underestimated, while height was overestimated, compared to direct measures. With all the correction models, the corrected measures were closer to the direct measures. Prevalence rates for excess weight, calculated by selfreport measures, were underestimated by $24 \%$ in men and by $28 \%$ in women; with corrections, the underestimation decreased to $8 \%$ and $10 \%$, respectively. The results showed moderate agreement for self-report measures and substantial agreement for corrected measures compared to direct measures. The use of correction equations for self-report data proved to be a useful method for producing more trustworthy estimates of prevalence of overweight and obesity in the general population, usually estimated from self-report measures of weight and height in population surveys.

Self Report; Obesity; Overweight; Prevalence; Body Mass Index

\section{Resumen}

Los objetivos fueron: comparar medidas autoinformadas de peso y estatura con medidas evaluadas; evaluar el impacto de estas discrepancias sobre el indice de masa corporal (IMC) y las prevalencias de sobrepeso y obesidad; y aplicar modelos de corrección de las medidas autoinformadas y evaluar el grado de mejoría de las medidas corregidas, producidas por el uso de esos modelos. Se realizó un estudio transversal evaluando a 4.151 adultos (18 a 60 años), participantes en el Estudio Epidemiológico São Paulo Megacity. Se propusieron y probaron modelos de regresión lineal estratificados por sexo para la corrección de las medidas autoinformadas. Para evaluar la concordancia, se usó el coeficiente de correlación intraclase para las medidas evaluadas, medidas autoinformadas y medidas corregidas, $y$ el coeficiente kappa para las categorías de clasificación del IMC. El peso autoinformado y el IMC resultantes fueron subestimados mientras la estatura fue sobrestimada, comparados con las medidas evaluadas. Con todos los modelos de corrección, las medidas corregidas se convirtieron en más cercanas a las medidas evaluadas. Las prevalencias de exceso de peso, cuando se calculan a partir de las medidas autoinformadas, estaban subestimadas en un $24 \%$ en hombres y un $28 \%$ en las mujeres; con las correcciones, la infravaloración disminuyó a un $8 \%$ y un 10\%, respectivamente. Se identificó una concordancia moderada para las medidas autoinformadas y una concordancia sustancial para las medidas corregidas, cuando se compararon con las medidas reales. El uso de ecuaciones de corrección para dados autoinformados se mostró un método útil para producir estimaciones más fidedignas de la prevalencia de exceso de peso y obesidad en la población general, generalmente estimadas a partir de medidas de peso y estatura autoinformadas en las encuestas poblacionales.

Autoinforme; Obesidad; Sobrepeso; Prevalencia; Índice de Masa Corporal

Recebido em 01/Abr/2018

Versão final reapresentada em 31/Jan/2019

Aprovado em 13/mar/2019 